Corrigendum

\title{
Corrigendum to "Comparison of the Proliferation and Differentiation Potential of Human Urine-, Placenta Decidua Basalis-, and Bone Marrow-Derived Stem Cells"
}

\author{
Chengguang Wu, ${ }^{1,2}$ Long Chen, ${ }^{3}$ Yi-zhou Huang, ${ }^{1}$ Yongcan Huang $\mathbb{D}^{4,4,6}$ \\ Ornella Parolini $\left(\mathbb{1},{ }^{7,8}\right.$ Qing Zhong, ${ }^{9}$ Xiaobin Tian $\mathbb{1}^{3},{ }^{3}$ and Li Deng $\left(\mathbb{1}^{1}\right.$ \\ ${ }^{1}$ Laboratory of Stem Cell and Tissue Engineering, State Key Laboratory of Biotherapy, West China Hospital, Sichuan University, \\ Chengdu 610000, China \\ ${ }^{2}$ Institute of Pathology and Molecular Pathology, University Hospital Zurich, Zurich 8091, Switzerland \\ ${ }^{3}$ Department of Orthopedics, Guizhou Provincial People's Hospital, Guiyang, Guizhou 550000, China \\ ${ }^{4}$ Shenzhen Engineering Laboratory of Orthopaedic Regenerative Technologies, Orthopaedic Research Center, Peking University \\ Shenzhen Hospital, Shenzhen 518036, China \\ ${ }^{5}$ Shenzhen Key Laboratory of Spine Surgery, Department of Spine Surgery, Peking University Shenzhen Hospital, \\ Shenzhen 518036, China \\ ${ }^{6}$ Department of Orthopaedics and Traumatology, The University of Hong Kong, Hong Kong SAR 999077, China \\ ${ }^{7}$ Centro di Ricerca E. Menni, Fondazione Poliambulanza Istituto Ospedaliero, Brescia 25124, Italy \\ ${ }^{8}$ Istituto di Anatomia Umana e Biologia Cellulare, Università Cattolica del Sacro Cuore Facoltà di Medicina e Chirurgia, \\ Roma 00168, Italy \\ ${ }^{9}$ Children's Medical Research Institute, University of Sydney, Sydney, New South Wales 2145, Australia
}

Correspondence should be addressed to Xiaobin Tian; txb6@vip.163.com and Li Deng; dengli2000@gmail.com

Received 23 January 2019; Accepted 30 January 2019; Published 10 March 2019

Copyright (c) 2019 Chengguang Wu et al. This is an open access article distributed under the Creative Commons Attribution License, which permits unrestricted use, distribution, and reproduction in any medium, provided the original work is properly cited.

In the article titled "Comparison of the Proliferation and Differentiation Potential of Human Urine-, Placenta Decidua Basalis-, and Bone Marrow-Derived Stem Cells" [1], there were the following errors:

(i) The first image (USCs) in the second row (passage 10) in Figure 1 was incorrect

(ii) There was a missing in-text citation for reference [15] in the legend of Figure 4

The corrected Figure 1 and the corrected legend of Figure 4 along with the figure itself are shown herein. 

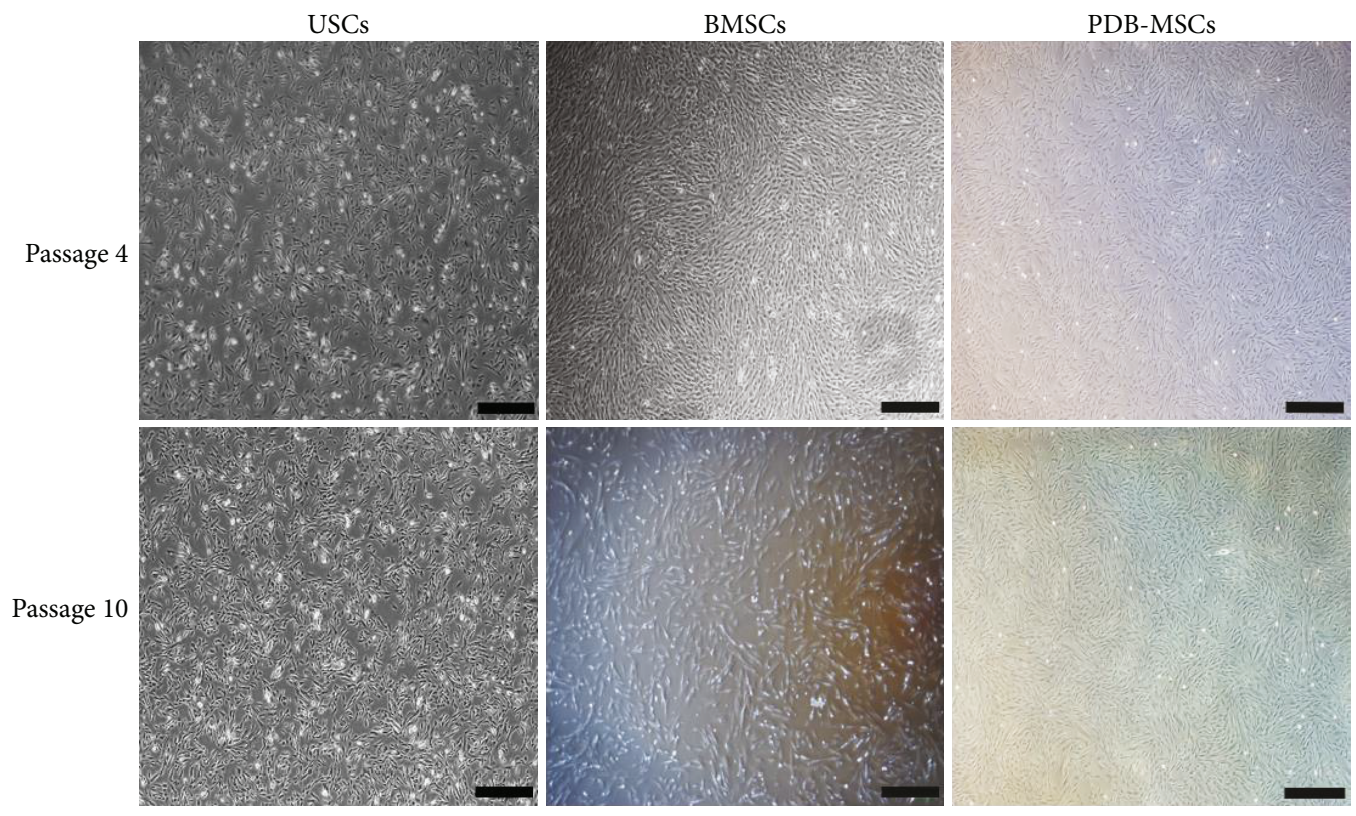

Figure 1: USC, BMSC, and PDB-MSC morphologies at passages 4 and 10. Scale bar $=500 \mu \mathrm{m}$. BMSCs: bone marrow-derived mesenchymal stem cells; PDB-MSCs: placenta decidua basalis-derived mesenchymal stem cells; USCs: urine-derived stem cells.
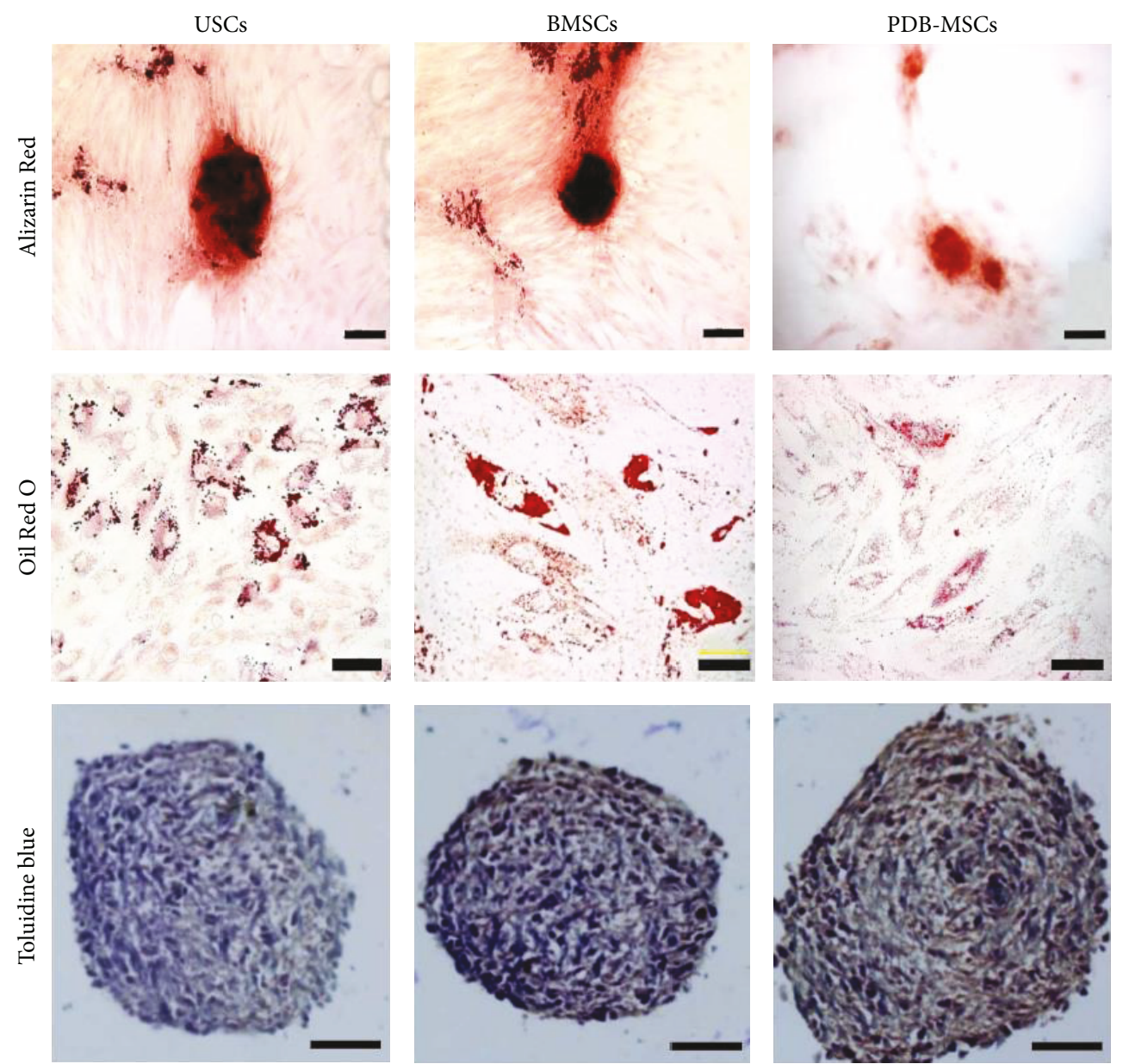

FIGURE 4: Osteogenic, adipogenic [15], and chondrogenic differentiation of USCs, BMSCs, and PDB-MSCs stained with Alizarin Red (scale bar $=100 \mu \mathrm{m}$ ), Oil Red O (scale bar $=20 \mu \mathrm{m})$, and Toluidine Blue (scale bar $=50 \mu \mathrm{m})$, respectively. BMSCs: bone marrow-derived mesenchymal stem cells; PDB-MSCs: placenta decidua basalis-derived mesenchymal stem cells; USCs: urine-derived stem cells. 


\section{References}

[1] C. Wu, L. Chen, Y. Z. Huang et al., "Comparison of the proliferation and differentiation potential of human urine-, placenta decidua basalis-, and bone marrow-derived stem cells," Stem Cells International, vol. 2018, Article ID 7131532, 11 pages, 2018. 


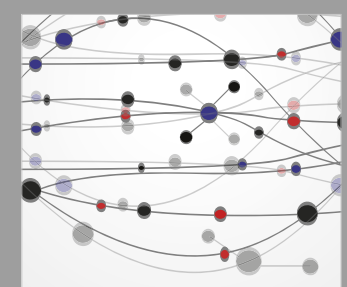

The Scientific World Journal
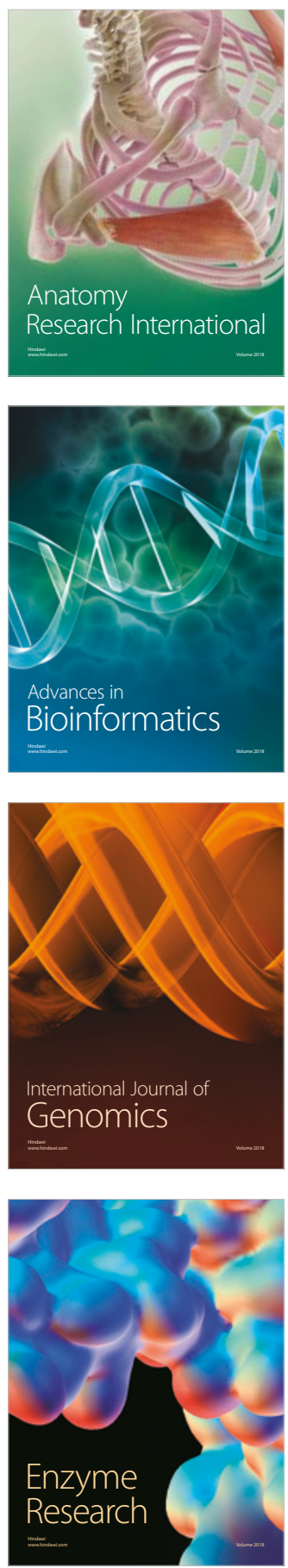
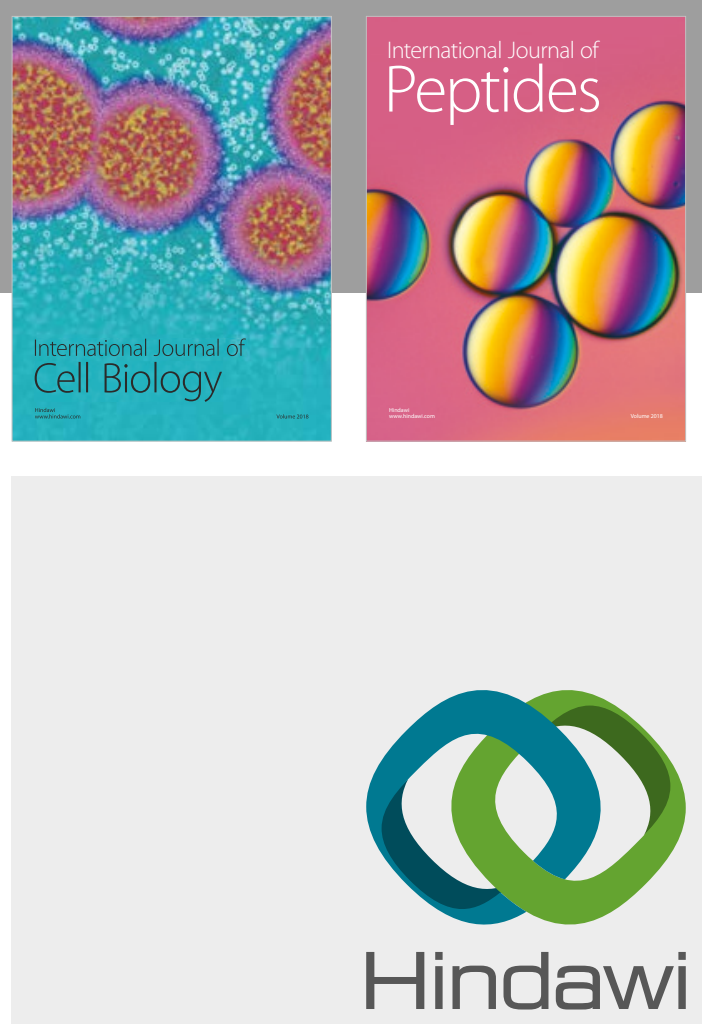

Submit your manuscripts at

www.hindawi.com
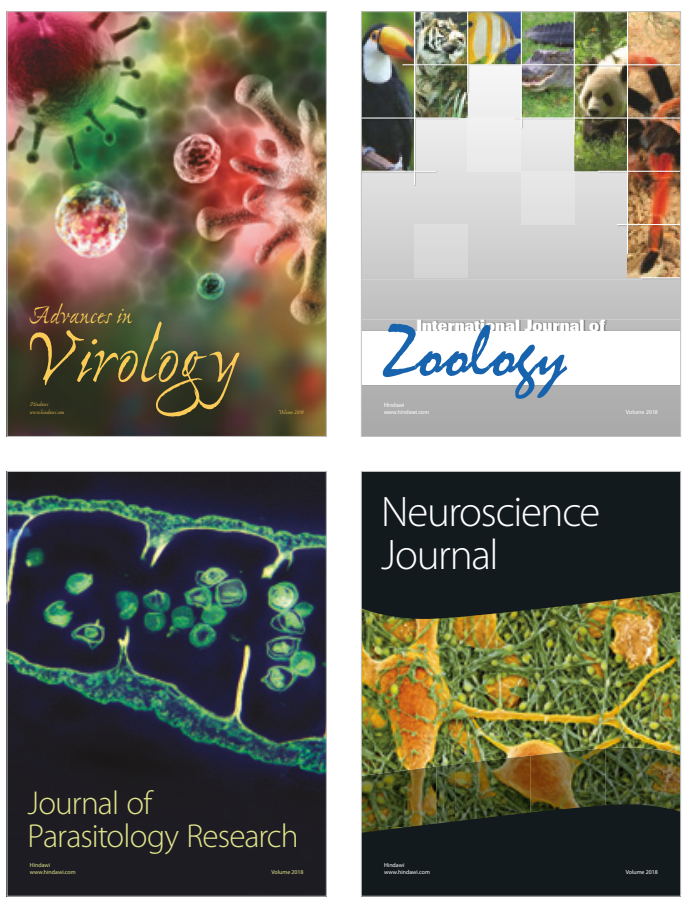
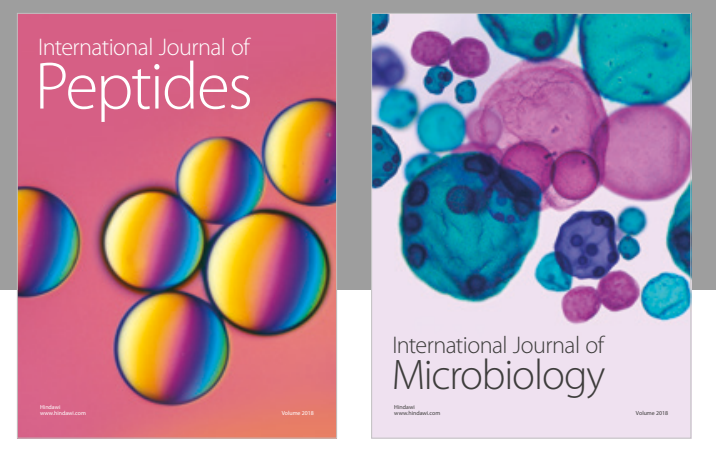

nternational Journal of Microbiology
Journal of
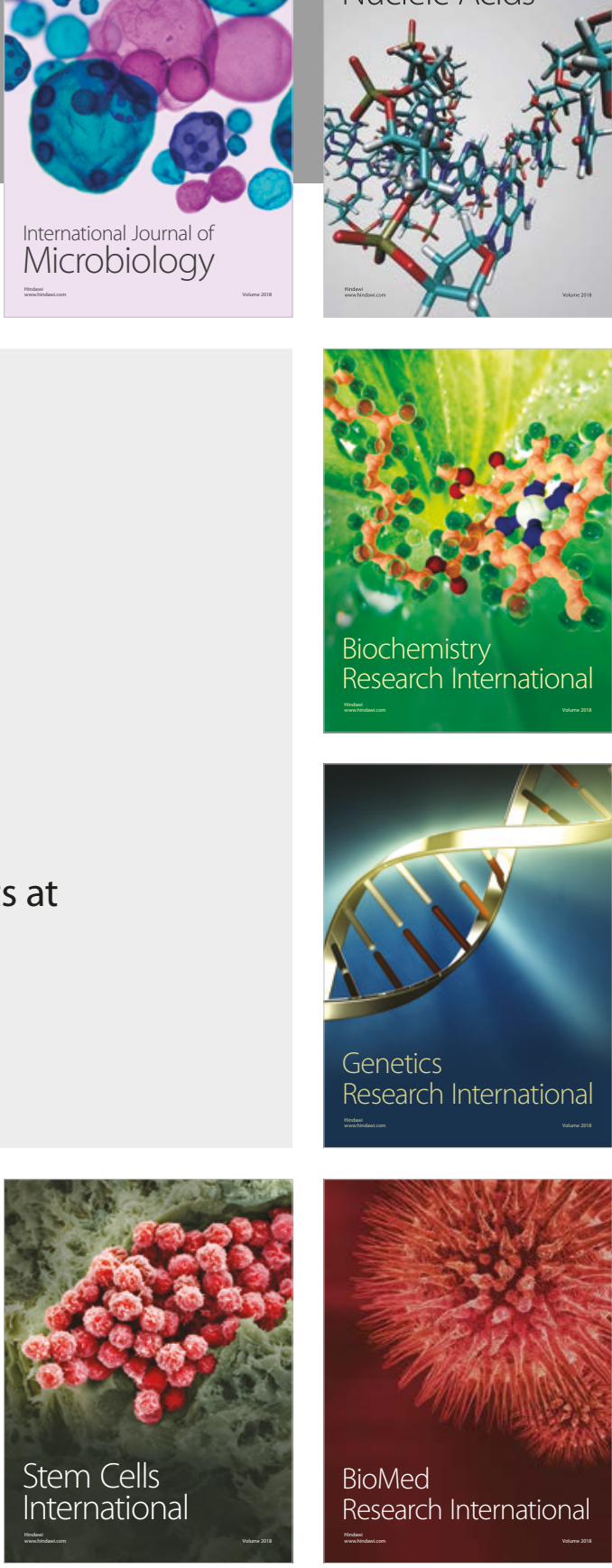
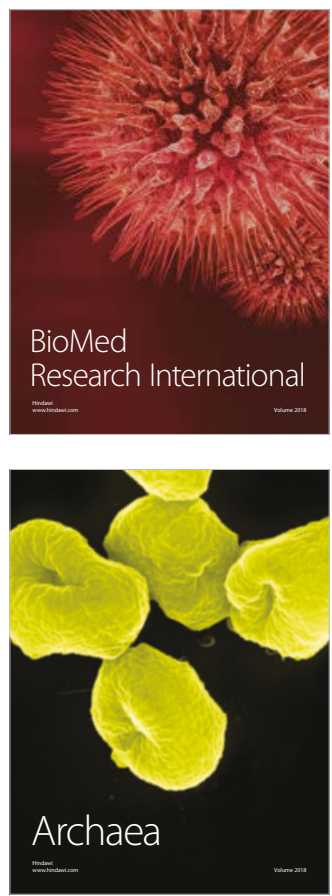